\title{
Development of a Minimalistic Pneumatic Quadruped Robot for Fast Locomotion
}

\author{
Kenichi Narioka, Andre Rosendo, Alexander Sproewitz and Koh Hosoda
}

\begin{abstract}
In this paper, we describe the development of the quadruped robot "Ken" with the minimalistic and lightweight body design for achieving fast locomotion. We use McKibben pneumatic artificial muscles as actuators, providing high frequency and wide stride motion of limbs, also avoiding problems with overheating. We conducted a preliminary experiment, finding out that the robot can swing its limb over $7.5 \mathrm{~Hz}$ without amplitude reduction, nor heat problems. Moreover, the robot realized a several steps of bouncing gait by using simple CPG-based open loop controller, indicating that the robot can generate enough torque to kick the ground and limb contraction to avoid stumbling.
\end{abstract}

\section{INTRODUCTION}

Bipedal and quadrupedal locomotion outperforms wheeled locomotion when it comes to dealing with unstructured or unpaved lands, which corresponds for a great portion of our planet. These locomotion patterns, which are mainly present in mammals, have been proven to be not only useful on handling obstacles, but also very energy efficient [1] [2]. Unfortunately, the high adaptability of mammal locomotion is associated to a high degree of complexity on lower limbs, involving redundant muscles with different roles. Hence, the details associated with animal locomotion, including gait and muscle activation patterns, are still unknown. Although biologists [3] [4] have made great advances on trying to associate quadrupedal muscular activation pattern with joint angles, output force and even gait pattern, to date there is no robot capable of successfuly replicating quadruped behavior through a biomimetic approach.

In 1990, Raibert created a quadruped robot which could perform different gaits [5], being the predecessor for the BigDog [6], using the virtual leg principle to assure stability. On a similar line of development we can mention KOLT and Scout II [7] [8], which are also quadruped robots with some degree of stability during locomotion. These three robots, in spite of the fact that their locomotion is stable, can not be considered as biomimetic approaches when it comes to the limb morphology. The majority of quadrupeds is known for their 3-linked leg structure, such as lions, dogs, elephants and cheetahs. In such a structure the third link (metatarsus/metacarpus) joins the other 2 links to increase the range of motion. Robots such as Puppy and Cheetah

This work was partially supported by KAKENHI 23220004, KAKENHI 24000012 and KAKENHI 23700233.

The authors Kenichi Narioka, Andre Rosendo and Koh Hosoda are with the Department of Multimedia Engineering, Graduate School of Information Science and Technology, Osaka University, Japan. The author Alexander Sproewitz is with Biorobotics laboratory, Institute of Bioengineering, School of Engineering, Ecole Polytechnique Federale de Lausanne, Switzerland.

narioka@ist.osaka-u.ac.jp
(HRL) [9] [10] possess this 3-link structure, better depicting the reality observed on the biological systems. Morphological complexity is often associated with control complexity: Legs with more links require more muscles for activation, exponentially increasing the number of parameters, specially if redundant and biarticular muscles are involved, as studied in [11]. Aiming to minimise the complexity of control patterns while keeping the morphological richness from biological systems, robots such as Cheetah (EPFL) and Oncilla [12], [13], [14] tried to explore the 3-link system through a pantographic mechanism, allowing a minimalistic control of 3 joints with 2 electric motors.

Due to problems observed with overheating during fast locomotion on the Oncilla robot, in this paper we approach the same successful morphology with a fast-locomotion oriented actuation principle: pneumatic artificial muscles. Differently from electric motors, McKibben pneumatic artificial muscles can provide high frequency without increasing its temperature, being an excellent candidate for high speed locomotion [15], [16], [17], [18]. Our proposed quadruped robot named "Ken", depicted in Fig. 1, has pneumatic valves with which air muscles can be controlled. Similarly to its predecessor, Ken's air muscles allow hip flexion/extension and a leg shortening through a combined flexion of knee and ankle (elbow and wrist for forelimbs), being capable of reaching high stride frequencies with virtually no temperature increase.

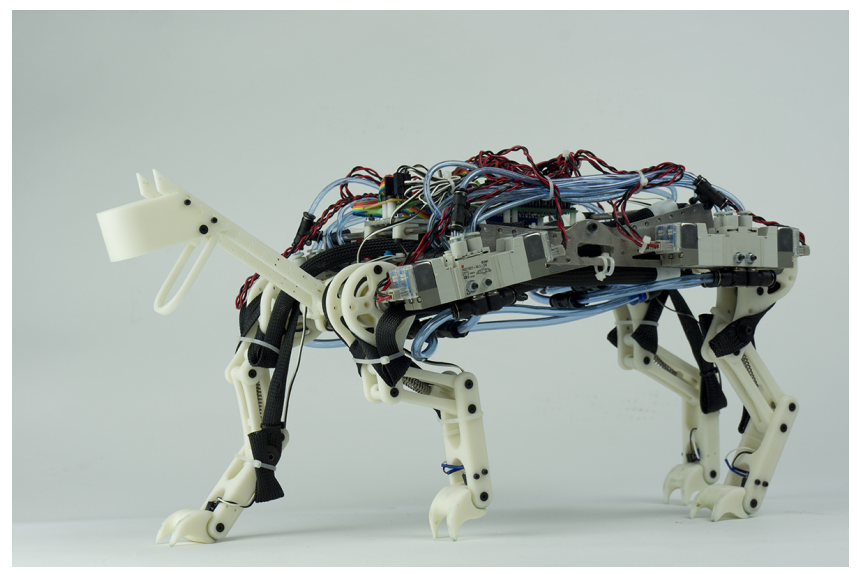

Fig. 1. Pneumatic quadruped robot "Ken". One of the main objective of this robot is to realize its fast locomotion utilizing the characteristics of the pneumatic artificial muscle. 

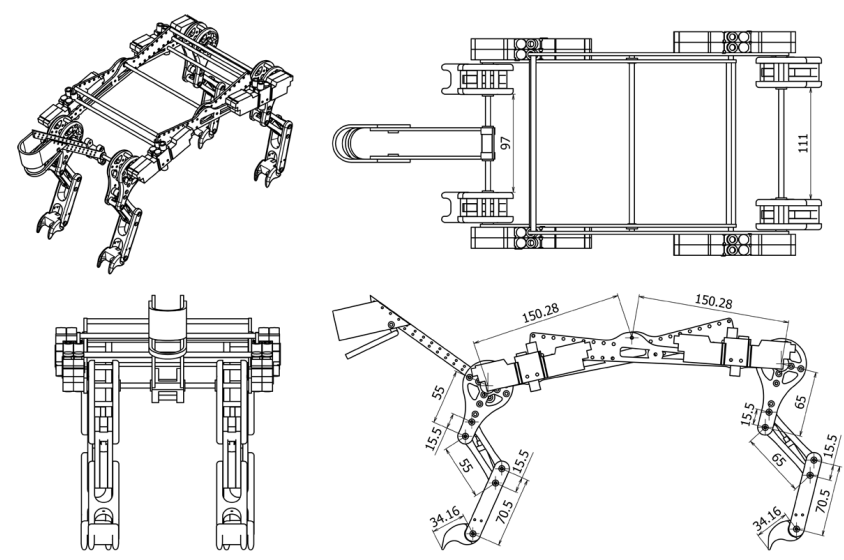

Fig. 2. Design of the quadruped robot "'Ken". Length of each limb is illustrated in the figure. The first and second proximal segments of a forelimb is relatively shorter than those of a hindlimb. The spinal pitch joint exist at the center between a forelimb and a hindlimb

TABLE I

KEN'S KEY CHARACTERISTICS

\begin{tabular}{c|c} 
Property & Value \\
\hline Length $\times$ Width $\times$ Height & $350 \times 225 \times 250 \mathrm{~mm}$ \\
\hline Total weight & $1.86 \mathrm{~kg}$ \\
\hline Forelimb weight & $0.07 \mathrm{~kg}$ \\
\hline Hindlimb weight & $0.07 \mathrm{~kg}$ \\
\hline Number of valves & 8 \\
\hline Number of muscles & 12 \\
\hline Number of touch sensors & 4
\end{tabular}

\section{Roвot DESIGN}

Fig.2 shows the design of the quadruped robot "Ken". Length, width, and height of the whole body excluding the ornamental head are around 350,225 , and $250 \mathrm{~mm}$, respectively. More detailed description of the length of each limb are shown in Fig.2, which are decided based on that of Oncilla [14]. Basic specifications of the robot is shown in Table I. Since the most of the parts of forelimb and hindlimb are made of acrylonitrile butadiene styrene created with 3D printing system (Dimension Elite, Stratasys Inc.), the weight of one limb is no more than 70 grams, which is significant characteristics for fast leg swinging. On the other hand, links of the trunk, that has to endure the high tension by pneumatic muscles are made of magnesium alloy (AZ31), which has a good balance of strength and lightweightness. The total weight of the robot is $1.86 \mathrm{~kg}$ including micro controller, battery, solenoid valves, actuators within its body. Although air is supplied from an external compressor so far, a compact cartridge liquid $\mathrm{CO}_{2}$ inside is available for a short period movement.

Skeletal structure of the robot is shown in Fig. 3. Each limb consists of four links and four joints, one of which is constrained by a pantograph mechanism, so that each limb has three degree of freedom $(\mathrm{DoF})$ : proximal joint's flexion/extension, distal joint's flexion/extension, and leg's

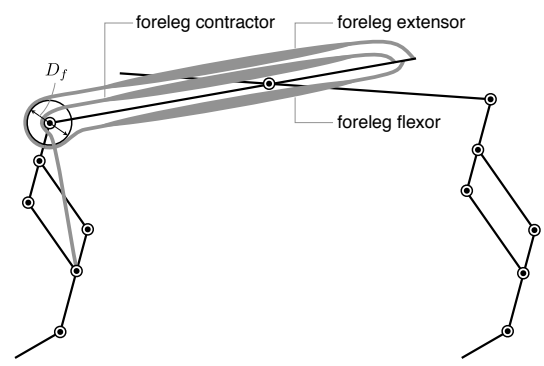

(a) foreleg

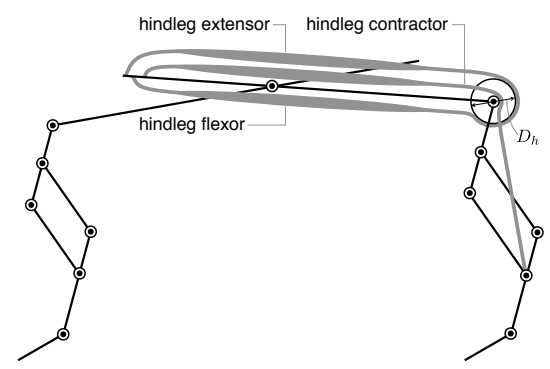

(b) hindleg

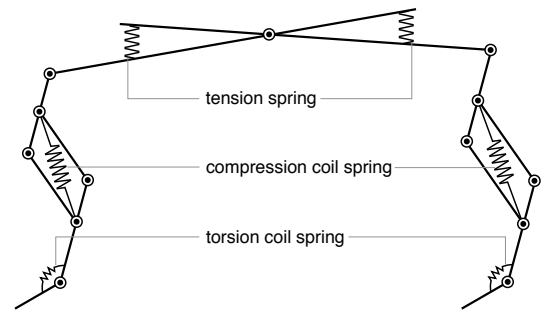

(c) passive elastic elements

Fig. 3. Skeletal structure and muscle arrangement of the "Ken". There are 13 DOF including a hinge joint of spine. Each limb, consisting of four links and four joints, one of which is constrained by a pantograph mechanism, has three degree of freedom (DoF): proximal joint's flexion/extension, distal joint's flexion/extension, and leg's contraction/extension. There are three muscles for each limb; extensor, flexor, and contractor as shown in (a) and (b). These muscles are responsible to a extension of the proximal joint, a flexion of the proximal joint, and a contraction of the leg, respectively. Passive springs are implemented at the spine joint, the distal joints, and pantograph mechanisms as shown in (c).

contraction/extension. There are totally 13 DOF including a hinge joint of spine, which are driven by McKibben pneumatic artificial muscles and passive elastic elements. The muscle is also lightweight (around 10 grams/muscle) since it consists of an inner rubber tube and an outer nylon sleeve. The length and stiffness of the muscle changed when compressed air is supplied to the inner tube. Since the contraction ratio is up to $25 \%$, it is important to use as long muscle as possible. We carefully designed an arrangement of muscles as illustrated in Fig. 3. There are three muscles for each limb; extensor, flexor, and contractor. The moment arm of the extensor and the flexor of forelimb and hindlimb are determined by the diameter of pulleys $D_{f}$ and $D_{h}$, respectively. On the other hand, the moment arm of the contractor is set to be very small so that it shortens the limb length with little influence on the extension and the flexion. 
There are two solenoid valves ( 5 ports and 3 positions type, VQZ1321, SMC Co.) for each limb, one of which supplies compressed air to both an extensor and a flexor. The other valve supplies compressed air to a contractor. Passive elastic elements are used as shown in Fig. 3(c). There is touch sensor on the distal link of each limb, which can detect a contact to the ground.

\section{EXPERIMENT}

In order to achieve fast locomotion of the robot, it is necessary to realize high frequency and wide stride oscillation of limbs. We conducted a preliminary experiment to verify whether high frequency motion and sufficient angular amplitude is compatible in the developed robot.

Experimental environment is illustrated in Fig. 4. The trunk is mechanically fixed to a steel frame. The right hindlimb is chosen to be extended and flexed periodically by sending periodic signal from micro controller to its solenoid valve. State A, supplying air to the extensor and exhausting air from the flexor, and state B, supplying air to the flexor and exhausting air from the extensor, of the valve is switched alternately, the cycle of the switching is represented as $T / 2[\mathrm{~ms}]$. Frequency $f$ of the limb swinging becomes $f=1 / T[\mathrm{~Hz}]$. Air pressure supplied from the external compressor is set to be $0.5 \mathrm{MPa}$. Moment arm of hip joint $D_{h}$ is $30 \mathrm{~mm}$. The motion of the limb is recorded with 600 fps by high speed camera (FX1, Casio Computer Co., Ltd).

An example of the movement is shown in Fig. 5, in which the period $T=220 \mathrm{~ms}$, then $f=4.55 \mathrm{~Hz}$. We changed the frequency of the oscillation, measuring the maximum and minimum hip angle $\theta_{h}$. The result is shown in Fig.6. The horizontal axis represents the period $T$ and the vertical one represents the angular amplitude of the oscillation. Maximum value and minimum value are connected with a straight line for each parameter $T$. While the period is higher than 200 $\mathrm{ms}$, angular amplitude is more than $130 \mathrm{deg}(\max >+67$, $\min <-64$ ). Although the amplitude tends to decrease with decreasing of the period, it is noticeable that the amplitude of extension (negative hip angle) has peak around $T=130 \mathrm{~ms}$. It seems to be due to the resonance effect, which might be helpful for achieving a balance between high frequency and wide stride. The reason why the maximum values keep around $67 \mathrm{deg}$ while $T>130 \mathrm{~ms}$ seems due to the limb's physical contact to the trunk.

As another preliminary experiment, we have tried to make the robot run on the treadmill with CPG-based open-loop controller referring to [19], [20]. All muscles are activated periodically, while the activation pattern of the right forelimb and the left forelimb is set to be the same, and also that of the right hindlimb and the left hindlimb is set to be the same. Tuning the phase lag between the forelimb and the hindlimb, the robot realized a bouncing gait of several steps shown in Fig. 7. So far the running gait is not so stable, it performed not only sufficient swing limb frequency and wide range of motion but also an enough torque generation to kick the ground and a limb contraction to avoid stumbling.

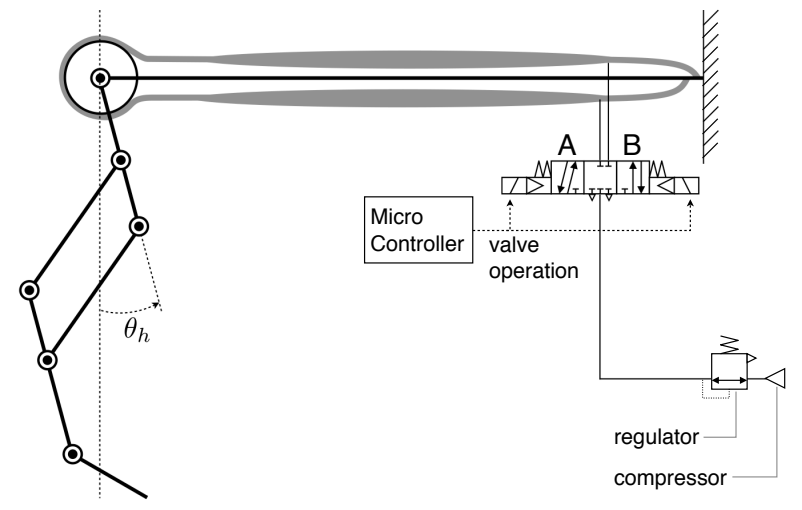

Fig. 4. Experimental setup. The trunk is mechanically fixed to the environment. The right hindlimb is extended and flexed periodically by sending periodic signal from micro controller to the solenoid valve. State A and state $\mathrm{B}$ of the valve is switched alternately at $T / 2[\mathrm{~ms}]$ intervals. Frequency $f$ of the limb swinging becomes $f=1 / T[\mathrm{~Hz}]$. Air pressure supplied from the external compressor is set to be $0.5 \mathrm{MPa}$. Moment arm of hip joint $D_{h}$ is $30 \mathrm{~mm}$.

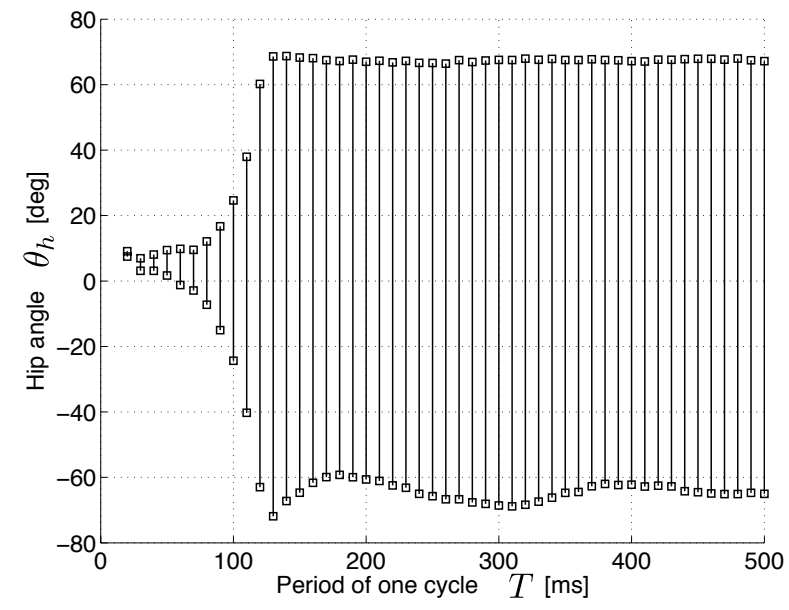

Fig. 6. Relationship between period and range of motion. The horizontal axis represents the period $T$ and the vertical one represents the angular amplitude of the oscillation. Maximum value and minimum value are connected with a straight line for each parameter $T$. While the period is higher than $200 \mathrm{~ms}$, angular amplitude is more than $130 \mathrm{deg}(\max >+67$, $\min <-64)$.

\section{CONCLUSION}

In this study, we described the design of the pneumatic quadruped robot "Ken". McKibben pneumatic artificial muscles are arranged in its body, which induce the limb movement with high frequency and wide stride without an overheating problems. We investigated the relationship between the frequency and the range of motion of a hindlimb to find out that it can achieve high frequency while keeping wide range of motion. Moreover, the robot realized a several steps of bouncing gait by using CPG-based open loop controller. It indicates that the robot has enough torque generation to kick the ground and limb contraction to avoid stumbling. In the near future, we will tackle problems such as fast locomotion, adaptive locomotion and gait transition, using the developed robot as a platform. 

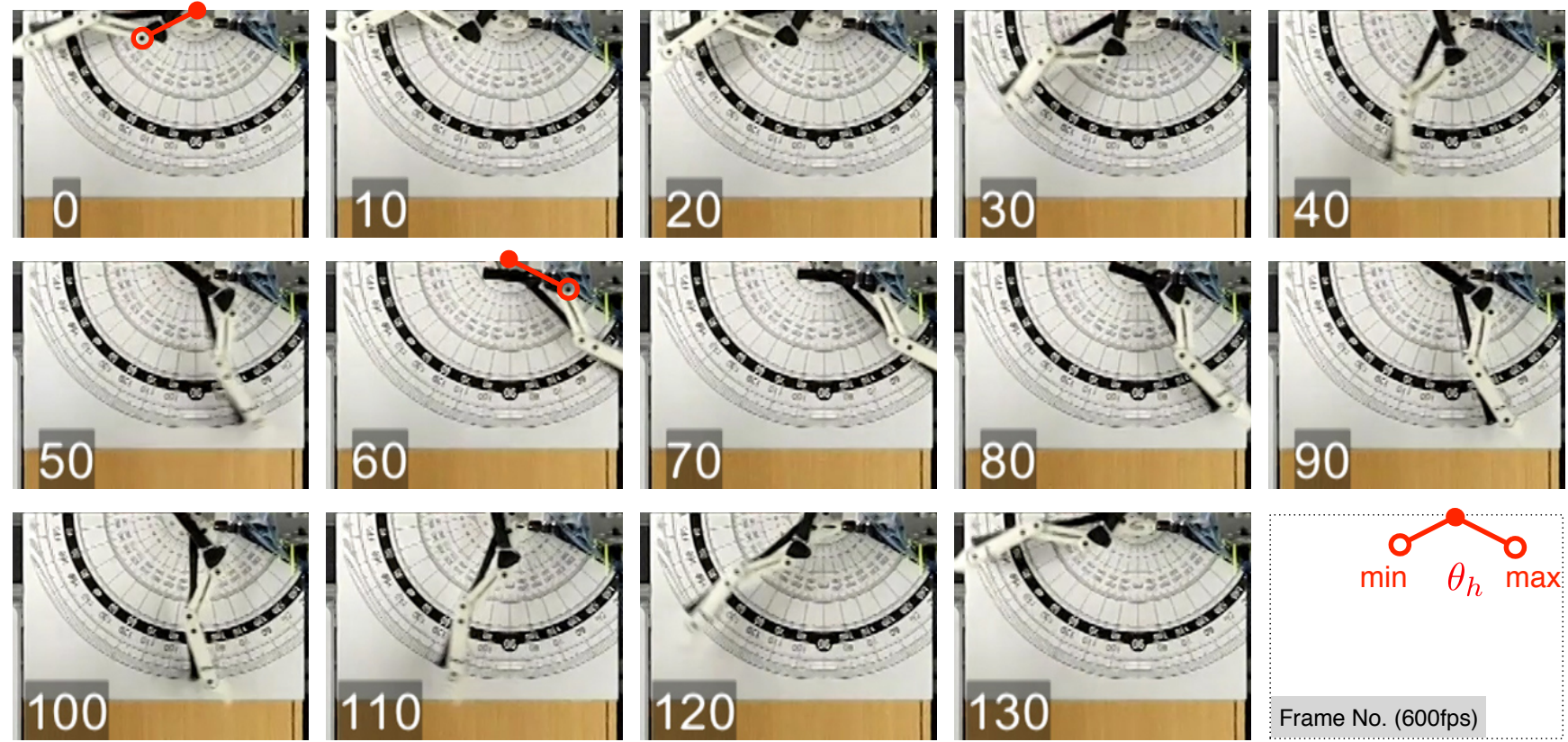

Fig. 5. Movement of the right hindlimb within one cycle while the period $T=220 \mathrm{~ms}$. The maximum and minimum values of hip angle $\theta_{h}$ was measured. In this example, $\theta_{h}$ got the maximum around $100 \mathrm{~ms}$ (Frame No. 60) and the minimum around 0 ms (Frame No. 0).
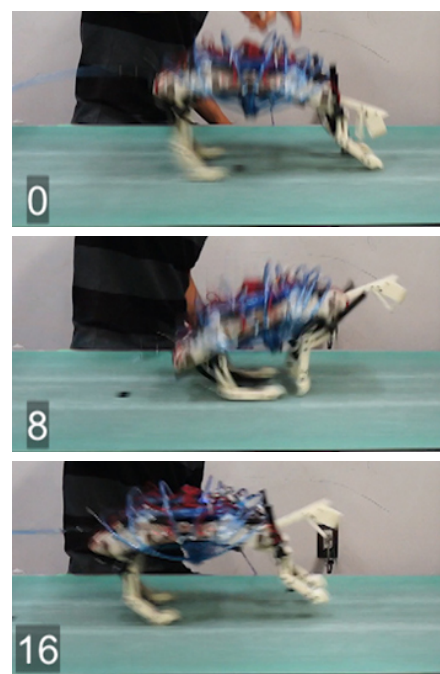
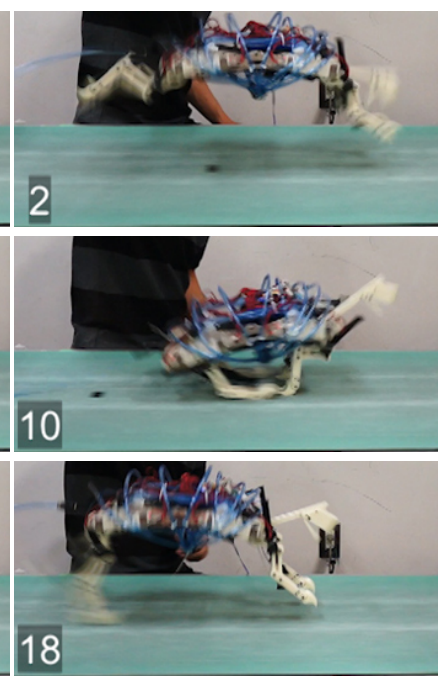
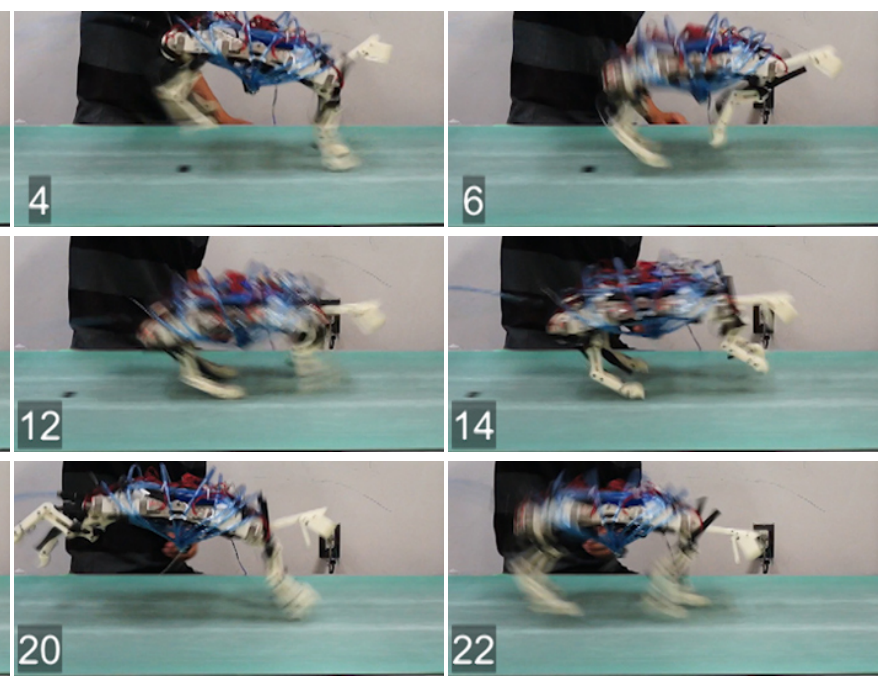
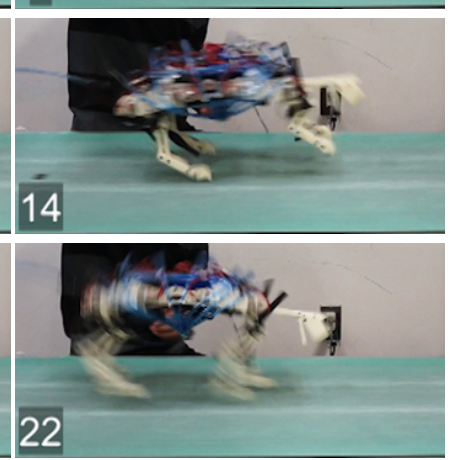

Frame No. (30fps)

Fig. 7. Running behavior of "Ken". The robot run on the treadmill with CPG-based open-loop controller. All muscles are activated periodically, while the activation pattern of the right forelimb and the left forelimb is set to be the same, and also that of the right hindlimb and the left hindlimb is set to be the same.

\section{REFERENCES}

[1] T. McGeer, "Passive dynamic walking", J. Robot. Res., Vol. 9, 1990, pp. $62-82$.

[2] Steven H. Collins, Andy Ruina, Russ Tedrake, and Martijn Wisse, "Efficient bipedal robots based on passive-dynamic walkers", SCIENCE, Vol.307 pp.1082-1085, 2005.

[3] I. Engberg and A. Lundberg, "An electromyographic analysis of muscular activity in the hindlimb of the cat during unrestrained locomotion", Acta Physiol. Scand., vol. 75, 1969, pp. 614-630.

[4] B.I. Prilutsky, W. Herzog and T. Leonard, "Transfer of mechanical energy between ankle and knee joints by gastrocnemius and plantaris muscles during cat locomotion", J. Biomech., vol. 29, 1996, pp. 391403.

[5] M.H. Raibert, "Trotting, pacing and bounding by a quadruped robot", J. Biomech., Vol. 23, 1990, pp. 79-98.

[6] M. Raibert, K. Blankespoor, G. Nelson, R. Playter, and T. B. Team., "Bigdog, the rough-terrain quadruped robot", In International Conference of Automatic Control World Congress, 2008.

[7] K. Waldron, J. Estremera, P. Csonka and S. Singh, "Analyzing bounding and galloping using simple models", J. Mech. and Robot., Vol. 1, 2009 
[8] I. Poulakakis, J. Smith and M. Buehler, "Modeling and experiments of untethered quadrupedal running with a bounding gait: the scout II robot", J. Robot. Res., Vol. 24, 2005, pp.239-256.

[9] K. Aschenbeck, N. Kern, R. Bachmann and R. Quinn, "Design of a quadruped robot driven by air muscles" in Proc. Intl. Conf. Biomed. Robot. and Biomechatronics, 2006, pp 875-880.

[10] M. Lewisy, M. Buntingy, B. Salemi and H. Hoffmann, "Toward ultra high speed locomotors: design and test of a cheetah robot hind limb", in Proc. Intl. Conf. on Robotics and Automation, 2011, pp 1990-1996.

[11] K. Hosoda, Y. Sakaguchi, H. Takayama and T. Takuma, "Pneumaticdriven jumping robot with anthropomorphic muscular skeleton structure", Auton. Robot., vol. 28, 2010, pp 307-316.

[12] S. Rutishauser, A. Sproewitz, L. Righetti and A. J.Ijspeert, "Passive compliant quadruped robot using central pattern generators for locomotion control", in Proc. Intl. Conf. Biomed. Robot. and Biomechatronics, 2008, pp 798-803.

[13] Witte, H., R. Hackert, K.E. Lilje, N. Schilling, D. Voges, G. Klauer, W. Ilg, J. Albiez, A. Seyfarth, D. Germann, M. Hiller, R. Dillmann, and M.S. Fischer, "Transfer of biological principles into the construction of quadruped walking machines". in Proc. of the Second International Workshop on Robot Motion and Control,pp. 245-249, 2001

[14] A. Sproewitz, A. J.Ijspeert et al., "Oncilla robot : a light-weight bioinspired quadruped robot for fast locomotion in rough terrain", in Proc. Fifth Intl. Symp. Adaptive Motion on Animals and Machines, Osaka, Japan, 2011.

[15] D. Caldwell et al. "Control of pneumatic muscle actuators", IEEE Control Systems, pp.40-47, 1995.

[16] R. van der Linde, "Design, Analysis, and Control of a Low Power Joint for Walking Robots by Phasic Activation of McKibben Muscles", IEEE Transactions on Robotics and Automation, Vol.15, No.4, pp.599604, 1999.

[17] Ryuma Niiyama, Yasuo Kuniyoshi, "Pneumatic Biped with an Artificial Musculoskeletal System", Proceedings of 4th International Symposium on Adaptive Motion of Animals and Machines, pp.8081,2008 .

[18] Kenichi Narioka, Koh Hosoda, "Designing Synergistic Walking of a Whole-Body Humanoid driven by Pneumatic Artificial Muscles: An empirical study", Advanced Robotics, Vol.22 No.10, pp.11071123,2008

[19] A. Sproewitz, R. Moeckel, J. Maye, A. Ijspeert, "Learning to Move in Modular Robots using Central Pattern Generators and Online Optimization", The International Journal of Robotics Research, Vol. 27, pp.423-443, 2008

[20] Kenichi Narioka, Koh Hosoda, "Motor Development of an Pneumatic Musculoskeletal Infant Robot", IEEE International Conference on Robotics and Automation, pp.963-968, 2011 